Department of Health and Human Services

Office of the Secretary

Office of Assistant Secretary for Health

Office of Population Affairs

Washington, DC 20201

opa@hhs.gov

Copies to:

michael.lauer@nih.gov

operasystemspolicy@nih.gov

sciencepolicy@mail.nih.gov

michelle.bulls@nih.gov

francis.collins@nih.gov

January 27, 2021

Dear Mr. Secretary:

I have spent decades on improving medicine and spent last 5 years to study cancer and chronic diseases.

In the attached article intended for Congress and state legislatures, I prove that medicine is deeply flawed, treatment options are extremely narrow, and treatments are not unable to address multiple cause factors. Due to flaws, money influences, biases, and intention to preclude real cures, medicine is unable to use the biggest cure for COVID-19. Temperature is a factor responsible for 100 times higher incidence and death rates. Ventilation condition in existing buildings in the U.S. is the first risk factor in schools, universities, office buildings, etc. The proposed measures can completely control the pandemic, solve medical spending shortage, revive national economy, and find cures for chronic diseases.

I can be reached at 202-560-3000 or tempaddr2@atozpatent.com.

Sincerely yours,

Jianqing $W u$

Jianqing $\mathrm{Wu}$, Ph.D., J.D.

Healthier World

Healthier World, FHSC v1.00 


\title{
Essential Measures for Ending COVID-19 and Other Pandemics Congress Should Establish True Health Science
}

\author{
Jianqing Wu, Ph.D., J.D. \\ Healthier World
}

This article is written for the U.S. lawmakers, state lawmakers and local government officials concerning flaws and problems in medicine, most powerful measures for ending the COVID-19 pandemic, and what the U.S. should do address the catastrophic health crisis which will inevitably be in front of the U.S. government and state governments.

\section{A. Medicine Misses the Biggest Cure for COVID-19}

COVID-19 is a disease that is distinctively associated with low temperature. It is number-one or number-two factor next to age. Temperature affects incidence and death rates always as a key contributory factor hidden in lifestyle, working habit, and cultural activities.

The death rates for any nation between winter and summer differs by 10 to 20 folds (data not shown, See data from www.worldometers.info). This difference pattern can be seen for nearly all nations. Temperature is number-one influencing factor within U.S. and nations including Japan, Singapore, Thailand, Vietnam, Taiwan, and Laos. The common pattern shows that temperature is a super strong factor affecting pandemic outcomes.

I try to find what causes great differences in incidence and death rates between different nations.

Great Differences in Incidence Rate and Death Rate Between Selected Western Nations and Selected Asian Nations

\begin{tabular}{|r|l|r|r|r|}
\hline$\#$ & \multicolumn{1}{|c|}{$\begin{array}{c}\text { Western } \\
\text { Countries }\end{array}$} & $\begin{array}{c}\text { Tot Cases/ } \\
\text { 1M pop }\end{array}$ & $\begin{array}{l}\text { Deaths/ } \\
\text { 1M pop }\end{array}$ & \multicolumn{1}{c|}{ Population } \\
\hline 1 & Belgium & 58,898 & 1,771 & $11,617,701$ \\
\hline 2 & Italy & 39,962 & 1,385 & $60,412,161$ \\
\hline 3 & UK & 51,491 & 1,370 & $68,084,411$ \\
\hline 4 & USA & 75,287 & 1,252 & $332,082,117$ \\
\hline 5 & Bulgaria & 30,843 & 1,250 & $6,919,124$ \\
\hline 6 & Hungary & 36,869 & 1,214 & $9,646,640$ \\
\hline 7 & Spain & 51,584 & 1,168 & $46,764,883$ \\
\hline 8 & Switzerland & 58,358 & 1,029 & $8,689,789$ \\
\hline 9 & France & 45,370 & 1,096 & $65,354,019$ \\
\hline
\end{tabular}




\begin{tabular}{|c|l|r|r|r|}
\hline$\#$ & \multicolumn{1}{|c|}{$\begin{array}{c}\text { Western } \\
\text { Countries }\end{array}$} & $\begin{array}{c}\text { Tot Cases/ } \\
\text { 1M pop }\end{array}$ & $\begin{array}{c}\text { Deaths/ } \\
\text { 1M pop }\end{array}$ & \multicolumn{1}{c|}{ Population } \\
\hline 10 & Sweden & 53,576 & 1,078 & $10,134,279$ \\
\hline & Asian & & & \\
\hline 1 & Countries & 7,648 & 110 & $1,387,530,727$ \\
\hline 2 & India & 3,459 & 99 & $275,127,290$ \\
\hline 3 & Philippines & 4,599 & 92 & $110,388,276$ \\
\hline 4 & Pakistan & 2,361 & 50 & $223,256,147$ \\
\hline 4 & Bangladesh & 3,202 & 48 & $165,599,479$ \\
\hline 6 & Japan & 2,691 & 37 & $126,259,973$ \\
\hline 7 & S. Korea & 1,441 & 26 & $51,293,799$ \\
\hline 8 & Hong Kong & 1,310 & 22 & $7,530,759$ \\
\hline 7 & Malaysia & 5,294 & 20 & $32,595,582$ \\
\hline 9 & Singapore & 10,081 & 5 & $5,875,898$ \\
\hline 10 & Thailand & 183 & 1 & $69,897,587$ \\
\hline 11 & Vietnam & 16 & 0.4 & $97,824,681$ \\
\hline 12 & Taiwan & 37 & 0.3 & $23,840,809$ \\
\hline 13 & Laos & 6 & & $7,333,942$ \\
\hline
\end{tabular}

I consider data quality for Asian nations, but studies found countries with good epidemiological data of stroke are Hong Kong, Taiwan, South Korea and Singapore. In these countries, strokes rank as the second or third leading causes of death in the population. India data may be less reliable due to its huge population. At this stage, reporting COVID-19 cases by the internet does not require complex technologies and expensive resources. If a nation does track cases, it should be able to get fair estimates. In addition, pandemic conditions in different nations can be checked by reading patient stories. Taiwan is known for having a good universal healthcare system. Data from Japan and Taiwan can evaluate to justify reasonable reliability of the data from other nations.

Death rates in the selected Western nations are at least 10 times of those in the selected Asian nations, but can be higher than 100 times. Incidence rates also differ by more than 10 times. I want to find what causes the big difference between Western nations and Asian nations. I suspect that most of the observed differences between different nations are also caused by temperature. Population density is clearly not a strong factor. I do not believe that genetics or races cause the difference because Asian immigrants appear to be more susceptible to the virus. While personal diet is a big influencing factor on a personal basis, this effect tends to drop out in the population data because people use different diets. Work stress and population density cannot explain Japanese data. Even in Japanese data, incidence rates differ by more than 10 times between summer and winter. This again shows that temperature is the biggest factor. 
I found that what will not drop out from the population data are things and factors that are distinctly associated with temperature. I looked into lifestyle, culture-related activities, daily commuting rituals, work environments, working places, public places temperatures in light of temperature. I found that those factors are distinctly associated with exposures to temperature and cold air. People in the U.S. experience unique temperature fluctuations and cycles. If I go out to shop for one day, I can identify hundreds of scenarios where people screw up. I note that people in different parts of nation experience different patterns of temperature daily.

I note that several factors such as lack of physical activity, sedentary lifestyle, etc. are strong factors that cannot drop out of the population data, and must be contributory factors for the differences between nations. However, I believe that the effects of temperature can still override those factors. In other words, by avoiding exposure to low temperature, people can still avoid infection and death. Lack of physical activity and sedentary lifestyle lower the threshold at which lower temperature plays its role.

In the U.S., a small number of people may have severely compromised immune system. For this reason, I cannot say all COVID-19 diseases can be prevented. I imagine that the disease is caused by two kinds of temperature effects: within-culture effect of temperature and between-culture effect of temperature. The incidence rates can increase by at least 10 folds in winter within a culture. There are great differences in incidence and death rates between U.S. and the Asian nations. If the U.S. residents adopted Asian lifestyle and environment, the incidence rate would be reduced. This is caused by between-culture effects of temperature. Hypothetically, I imagine the U.S. residents lived the Japanese lifestyle, the incidence rate would be the baseline. By changing the lifestyle to the U.S. lifestyle, the incidence rate could increases by 5 folds. By exposure to temperature in winter, the (normalized) incidence rate could be increased by 10 folds. Thus, temperature is responsible for 50 times of increase. By avoiding all adverse effects of temperature, the incidence and death rates in U.S. could be reduced to $2 \%$. That is why I believe that $97-98 \%$ deaths can be avoided by proactively managing body temperature.

In my estimate, I use a large discounter to take into account the effect of foods, work stress, etc. that cannot drop out of the population data. The 10 folds for within-culture effect is a conservative number, and the 5 folds for betweenculture effect is also a conservative number. I can argue that even the cases in Thailand, Vietnam, Taiwan, and Laos can be attributed to temperature. All infection cases can be traced to temperature effect. I use $2 \%$ to take into account those individuals whose immune systems are severely compromised. Such individuals cannot be among those can live decades lives.

In a study, the authors found that temperature is number-one or numbertwo factor that affects the COVID-19 pandemic. Temperature is even more important than the government's first action and pandemic measures. Many studies concerning cold and flu similarly support this finding. I further found that 
temperature affects disease outcomes by different ways in different cultures. Every act, move and activity of each person in each day is under the influence of temperature. Temperature affects Japaneses and Americans by different ways. Temperature affects residents in California, Texas, and Maine in different ways. Temperature is always a determinant factor hidden in human daily activity.

While massive researches have found the decisive role of temperature, medicine does not even suggest that people should learn how to manage body temperature as the first measure. I reviewed studies in the last century, and also find that temperature affect anti-viral mechanisms, the innate immune function, acquired immune function, and tissue damage repairing functions. I had no slightest difficulty to find that the best prevention and best cure is proactive body temperature management. I and Ping Zha proposed a proactive body temperature management protocol, which lays the ground work and theory to manage body temperature as the primary measures to prevent and cure the disease. This work is rejected by Prepreint.org and SSRN.com. However, this article was able to see the light of day on both researchGate.net and an Open Science Foundation Server (https://osf.io/pcfna). My biggest "discoveries" were synthesis of a century of discoveries by hundreds to thousands of researchers.

It is obvious that the fastest and most effective measures for ending the pandemic is proactive temperature management, deep breathing exercise, and renovating ventilation systems for public buildings such as schools, universities, office buildings, and shoppers.

\section{B. Medicine Is Deeply Flawed Science}

Medicine misses absolutely the first prevention and first cure for the disease that could easily reduce $97 \%$ incidences and deaths. Medicine is an art that is deeply flawed in all aspects from research models and methods, practicing models, selection of treatment options, etc.

\section{A population-based reductionist static binary comparison model}

Anyone can see that health is a reflection of "mind-mediated personal multiple factor dynamic process balance". Thousands to millions of studies published after 1980 have produced conclusive evidence to show that:

(1) Population-based model is biased, inaccurate or even completely wrong as long as it is used to find cures for chronic diseases. Population based studies are only useful in finding potential causes and assessing the impact of factors, but have been routinely misused as treatment guidelines. If this method were used in car repair or plane repair, every car would be dead on road and every plane would crash.

(2) Reductionist treatment is poor at least, and completely wrong in most cases. Studies to examine the limitation of reductionist approach have proved in 
one way or the other that health cannot be addressed by focusing on one single factor. To overcome reductionist limitations, many biological sciences have evolved systems sciences such as systems biology to focus on the whole system.

(3) The binary scale is used in characterizing diseases, health, and a large number of health properties. This scale is used solely for convenience of research, but is contrary to reality of nature. The use of the scale introduces excessive errors (49.9\%) for most researches.

(4) Treatment effects are assessed by focusing on the body part only, while in reality, mind is often more important than the body. The role of mind was recognized in ancient medicines and noted in medicine 146 years ago. Post 1980 studies and recent studies also refute the notion that the body can be treated in isolation of mind.

(5) Static comparison is often improper because disease outcomes depend on the balance among multiple biological processes in a dynamic system. Process timing, activity phases, factor interactions, etc. often have decisive effects on health or disease outcomes. The difference from measuring A and B is often meaningless.

(6) The presumption that everything can be solved by mathematics is wrong and have no reality. Use of statistics, use of mathematical methods, etc were all wrong except in limited situations, where the effect of the factor under investigation is a super strong factor.

(7) Most disease theories in medical literature are grossly inaccurate or wrong. After massive disease causing factors are discovered, medicine fails to correct flaws in disease theories established before 1980. Somatic mutation theory, drug/toxicity independent action model, and epidemiological models are all very poor or completely wrong, depending on research purposes.

(8) Stressing the role of evidence is clearly unworkable in personalized medicine. Evidence acquired from a population has no relevance to any person, while evidence from a specific patient is very limited in reality. It is dangerous to rely upon partial evidence to make a treatment decision. Courts can rely on evidence purely for necessity, but reliance on partial evidence in medicine can cost lives. Concerning most health problems, what a doctor sees is not what really is. The multiple factor disease model makes evidence meaningless.

(9) The design drug concept started about 1980s, but the parallel researches in disease cause factors essentially refute its validity. With limited exceptions, the drug design principle cannot survive the firmly established multiple factors disease cause model. Chronic diseases are affected potentially by hundreds to thousands factors. The validity of the design drug concept is questioned because it fails to take into account tens to hundreds causal and influencing factors, mind-body interactions, tissue chemical environment, process dynamics, etc. If health can be achieved by focusing on a single target process, medicine can be reduced to Chemistry 101. 
In summary, a mountain of evidence acquired in the last half century has firmly proved beyond reasonable doubt that medicine is deeply flawed. If any health property has a value of 100 relative value, medical data, if used for treatment of diseases, may be off the mark by $90 \%-99 \%$. Some treatments may actually hurt patients. Many COVID-19 patients were harmed by misused drugs in an attempt to control body temperature. Given the massive foregoing problems, I cannot find any conceivable way to defend its validity. This is why medicine cannot cure chronic diseases.

\section{Medicine Has Become A Money Science}

Medicine has long become a money science. I mean that everything it does is to achieve highest revenues for interest groups. I will show how "medicine" meaning was changed and how medicine precludes real cures.

\section{Medicine Becomes an Art of Medicalization with Drugs}

"Medicine as cure" is an experienced-based notion that has stood longest time test. However, after human developed technology for making synthetic drugs, "medicines" was replaced by "synthetic drugs" without vetting the effects of synthetic drugs. Now drugs are used as the primary treatment options. The changed "medicines" clash with the evolution theory, mind-body theory, biological pathway network balance, etc. A single active compound can correct a large number of subtle changes caused by diseases in biological pathway networks. Drug benefits have been negated in a large number of studies on cancer, heart diseases, mental diseases, etc. Target drugs are based on the reductionist concept that a disease can be cured by correcting a single step in the biological pathway networks. It cannot happen except by accident.

Researches has produced hundreds of meta reviews, with most of them negating drug benefits. However, positive drug benefits in a small number of studies are inflated by clinical trials, flawed statistical analysis, flawed drug side effect model, flawed reductionist approach, flawed independent action model, and failure to consider organ redundant function. The true benefit-to-risk ratios are completely different from the nominal values of clinical trials. Numerous drug screening studies add more evidence that a good portion of drugs are carcinogens. This is why lifetime cancer risk has increased to 0.4 and will reach unity.

\section{Medicine precludes everything under the SUN, except revenue- generating objects.}

Half a century of researches has firmly established multiple factors disease cause model. Based on this reality, I find that medicine cannot address most health problems and chronic diseases. For diseases such as cancer, heart disease, or COVID-19 vulnerability, hundreds to thousands of factors can affect each person potentially. The first 10 cause factors on all patients of a same 
disease would be completely different. The most important contributory factors for John Doe must be different from those for Jack Doe even if they have the same genetic composition. Based on disease causes, 95\% of cancer cases can be prevented (as well as be cured), 95\% of heart diseases can be prevented and cured, I and Zha found that 97\% COVID-19 deaths could be prevented or avoided. AIDS researches imply that most infection cases can be prevented and effectively mitigated. Even though HIV is a virus, but lifestyle, dietary, social, environmental and political factors affect personal immune system.

\section{Medicine promotes less than $1 \%$ treatment options and precludes more than 99\% options including the best}

Medicine has become a junk science for an entirely different reason. Medicine has narrowly focused on drugs, devices, radiation and surgeries as primary treatment options. Medicine has precluded everything else under the Sun. It discriminates options from nature. All favored treatments are selected for their revenue-driving potentials. This means that nearly all clinical trials engage in chain-wise comparisons. Drug A is compared with drug B, then drug B is compared with drug $\mathrm{C}$, and drug $\mathrm{C}$ is compared with drug $\mathrm{D}$. Assuming that research method were valid, such studies can prove only relative merit of those drugs. As long as medical options are selected based on revenues, medical research cannot be a valid science because it is like choosing the tallest dwarf from a room full of dwarfs.

The research subjects and treatment options are further influenced by publishers. Medicine is only a money-science, such science cannot be scientifically valid. No one can claim honestly that any of studied treatment options is best, that drugs are better than air, temperature, laughter, etc, and chemotherapy is better than change of lifestyle. The article publishing practice further ruins scientific validity because publishers are biased. Money provides incentive to support more than two thousand drug trails for COVID-19, but provides no incentive for exploring the role of temperature. Medicine chooses drugs over temperature even though a century of studies prove that temperature is far more important than drugs. Influenced by money, medicine is unable to tell differences between a potential effective rate of $97 \%$ and potentially nothing.

\section{As a Money Science, Medicine Precludes All Real Cures.}

Medicine does not use specific criteria to preclude real cures using emotional state, exercise, lifestyle, diets, and avoidance. While studies have implied that real cures for chronic diseases are among those things, medicine also prevents them form being used as treatments in clinics.

\section{Research Standards Are Tailored to Inferior Revenue-Generating Options.}


While clinical trials are valid by approximate in studying strong treatments, they are deeply flawed in studying weak factors and treatments. Medicine has clung to clinical trials as the last ounce of validity evidence. By creating method as a final arbiter, medicine has artificially exaggerated scientific validity and suppressed any challenges and preclude real cures. Even though no reasonable mind can accept the notion that a research method is "good for everything", this notion has actually controlled medicine for nearly a century. This practice can continue controlling medicine because it is promoted by researchers, publishers, reviewers, doctors, and media. When the whole medical world accepts this practice, it creases a false impression of scientific validity is indisputable.

I and Ping Zha have proved that clinical trials are deeply flawed. We found that experimental approach is wrong if it is used to study weak effects of any factor or weak treatment. We have proved that an experiment cannot accurately determine the true effect of a weak factor if other factors affect the measured property. There is no way to resolve the contribution from the factor A and from contribution from factor B. In the last a few decades, researchers have produced massive evidence to prove that all health problems and diseases are influenced and controlled by a large number of weak factors.

While science must use experiments to gain insight to complex systems, one must understand that experiments from clinical trials may not be used as direct guidance in treatment of diseases. No single treatment protocol is good for all patients because no two patients are sufficiently similar in chronic diseases. Many non-medical problems cannot be evaluated by experiment. Many complex systems cannot be evaluated by experiment. For example, one cannot evaluate military performance by experiment.

\section{Money Has Turned The Medical System into a Dysfunctional System.}

A deeply flawed medicine has been manipulated by interest groups to achieve financial gains. All infrastructures in medicine are established to preclude real cures and disruptive discoveries:

(1) Medial publishers select articles by looking at their revenues potential. Publisher's decisions are mainly based on publish-for-money trade. The government can find a large number of scandals in the publishers' business. If an article can hurt readers but save millions of lives, the publisher will choose the option to keep their readers but let patients to die. This is strictly a business decision that no body can object. The world should have never trusted publication merit in the first place.

(2) The general acceptance standard is used in all aspects of medicine. This standard ensures that only commonplaces are published. Junk science in 30 s to 80s continue controlling medicine forever.

(3) The random drawing method used by publishers in selecting peers is an implied requirement that every statement in an article is acceptable and understandable to every potential peer. Thus, researchers must be extremely 
conservative in every aspect of researches. A safest bet is doing similar researches, using similar methods, and making limited findings.

(4) NIH funding practices are focused on researcher jobs, applicant's fairness with no focus on search impacts on cures, public health, etc. Public interest is only a slogan in NIH mission statements, but is replaced by a large number of technicalities and unnecessary limitations.

(5) Medical publishers use peer reviewers to kill disruptive, game-changing discoveries so that published articles will not disrupt their revenues. When a publisher assigns misfitted and incompetent reviewers, an expected review outcome is suppressing the article.

(6) Since most diseases can be traced to lifestyle, daily activities, work and thinking habits, and exposures, the best prevention and cures most probably come from patients who have fought diseases for decades. This was how most early treatments were discovered. However, medicine has completely precluded patient discoveries. No publisher accepts patient stories and treats patient stories as junk science. Publisher demands outrageous publication fees. Medicine, as both flawed and money science, has done everything to preclude all real cures from fork medicine, ancient medicines, personal discoveries, healing miracles, and disease avoiding experience.

Major publishers such as Nature, New England Journal of Medicine, and Science keep publishing junk science. If one draws 100 articles on clinical trials, I will prove all of them are deeply flawed. Their research subject compositions are revolved around money-generating objects and obsolete practices. Publisher's in-house review and peer review are established to stifle innovation and creating thinking. Biased reviewers may ignore critical discoveries but attack trivial technicalities. Their publishing practice is the most effective mechanism for maintaining flawed medicine perpetually.

\section{Flaws and Money Jointly Promote Fraud In Medicine.}

Medicine has failed for centuries, but interest groups promote it as "science" and "science-based medicine". Scientific validity is promoted by different people for different reasons. Some promotes medicine by honest mistake, some promotes flaws due to inability to challenge, some distort science by concealing knowledge, some kill true science by active suppression, and some misleads the public by willful fraud.

Most medical scientists were unaware of the flaws before 1980, and the early researchers made honest mistakes. Studies from 1980 to 2000 have produced evidence to question reductionist approach. However, a huge number of articles have been published to question the validity of clinical trials after 2000. Given the impacts of the research method, medical scientists should have explored and corrected noted flaws. Researchers failed to do their parts. Some are not in the position to challenge, some concern their own achievements, and some are afraid of losing funding. 
Given its failure, medicine has clung to clinical data as evidence of scientific validity. It is extraordinary to promote clinical trials as a gold research standard and the final arbiter of medical controversies. I found this practice is both absurd and fraudulent. When I entered laboratory on the first day, I was told that scientists were required to develop the best method for solving the problem under investigation. No other field of science has relied on a single research method as a final arbiter for resolving every dispute. Even most high school students could know that science is to explore systems, but not just acquire measurements. "Final arbiter" should be a proper term for court. The final arbiter has been manufactured for improper purposes by a small number of controlling persons and accepted by medical researchers who fail in thinking or integrity.

Different persons promote flawed models for different reasons. Drug companies and practicing doctors would need a gold standard to promote drug acceptability and shield legal liability. Drugs can attract patients by producing comparison data. If patients are injured by drugs, clinical data can shift drug injuries to patients. Public interest groups demand clinical trials in an attempt to prevent imminent danger without understanding the limitations. Some medical publishers demand experimental data from clinical trials. Even though research method is none of the publishing business, they use the gold standard to protect their client articles. By demanding clinical data, publishers can preclude anything that cannot be studied by using clinical trials and anything outside deeply flawed medicine. In addition, publishers use detail technicalities to prevent true and disruptive discoveries, but promote flawed reductionist researches. Publishers have killed disruptive researches and created a culture to promote money-driving articles, suppress disruptive discoveries, and preclude creative researches.

By using flawed research models, the interest groups have disciplined all aspects of medicine for decades. They control medicine mainly by affecting funding practices, publishing practices, and treatment practices. By controlling those key practices, interest groups have created a junk science landscape. By actively promoting clinical trials, medicine continues harming patients by falsely claimed scientific validity. Somatic mutation theory is clearly poor, but it is still used as the basis for cancer diagnosis and treatments. Epidemiological models were based on improper birth model, but are still used. Naturally, medicine precludes body temperature management as cures even though it is about 100 times more powerful than any drug. Medicine pays no attention to a large number of measures that can promote the immune system.

\section{E. Congress Cannot Rely on the Consensus of Experts in Medicine.}

The U.S. has relied on medicine for decades, but has reached a point the nation cannot solve medical spending. The problem has threatened national security and democratic process. Congress has tried to solve medical spending 
shortage by reallocating federal funds. Early efforts include Hillary Clinton's medial reform attempt in 1993 to the latest Affordable Care Act 2010. Hillary's universal health care for all Americans failed because its reform lacks the key ingredient to improve medical performance or the effort of finding cures for chronic diseases. For the same reason, Affordable Care Act 2010 must fail in light of expected huge financial burden from the COVID-19 pandemic and the coming health crisis. There is no conceivable likelihood that the U.S. government can meet needed medical spending. Those reforms failed because Congress did not go beyond medical science. Now, Congress must reject medicine as junk science.

Past medical reform efforts indicate that healthcare burden has reached to an unbearable point. By listening to the advice and consensus in medicine, Congress can ever solve this problem. The future medical spending is not $\$ 6$ trillion as once predicted. Past predictions must be changed to take into account four huge impacts of the COVID-19 pandemic. The first impact is that COVID-19 pandemic will increase the number of deaths in chronic diseases in the future. The financial burden from cancer, heart diseases, liver disease, kidney diseases, AIDS, mental diseases, etc. will be far more than what was predicted. Second impact is from degraded population health attributable to pandemic measures. With time passing, people are gaining weight, acquire bad life habits, pick up more harmful substances, and suffer emotional pain. The population will be much more vulnerable to COVID-19 disease and all other diseases. Patient stories indicate that survivors from the disease continue suffering health problems such as weakness, fatigue, lost taste and smell, lost lung functions, reduced physical strength, etc. Many are signs of permanent disabilities at different degrees. This is no longer a suspicion when independent studies show that the virus can damage Central Nervous System, lungs, heart, liver, and kidneys. The third impact comes from economical ruin. Most U.S. GDP may come from anti-pandemic measures. The $\$ 19$ trillion U.S. GDP does not reveal the serious financial ruining in education, restaurant, retail, travel, transportation, etc.

The fourth impact is potentially a catastrophe. Many studies found that the SARS-CoV-2 virus genetic composition is found in many types of immune cells. Whether the virus can permanently compromise human immune system cannot be answered for years. It is possible that some infected patients may acquire AIDS-like diseases many years later if they are unable to clear the virus completely. Mankind will fight against an air-transmittable AIDS in a big population. The ultimate failure of vaccines can be predicted from influenza vaccines. Vaccines can be defeated by viral mutation, compromised immune system, and adverse effect of temperature, while some patients may die from vaccine adverse reactions. A recently discovered problem is that vaccines cannot raise immune response to a sufficient strength to clear out the virus. Thus, vaccines can turn a patient into a virus development system, where the virus keeps mutation in an attempt to defeat the immune system's check. This patient can generate a huge number of mutations, may result in virulent viruses and 
transmit the viruses to others. If the world has millions to billions such systems, the stake is civilization continuation. No one can rule out this possibility.

The COVID-19 pandemic is not one that can be addressed by reallocating money. I must urge Congress to take measures to forestall this catastrophe before it comes. Like AIDS, the COVID-19 pandemic cannot be controlled by drugs and vaccines only, but must use systematic measures. Medicine cannot be obsessed to this less than 1\% options. Any further attempt to bind human hands should be viewed as crimes against humanity and civilization. Congress cannot rely on flawed money-driven medicine and consensus of experts who cannot think, are biased, or controlled by money.

Congress should see that the health problem is no longer a problem that can be solved by reallocating funds. A viable solution is establishing a valid medicine, creating a health culture, and training people with skills to avoid exposures. Without addressing those components, Congress can never successfully solve this financial crisis. I urge both democratic and republic lawmakers to come together to change legislative priority to find cures and cultivate a health culture.

Congress should use political wisdom in addressing this deeply flawed money-driven medicine. It should see that, any field, after it has been controlled by interest groups as revenue-generating system, cannot be a real science for the betterment of society. In other fields of sciences, Congress does not need to address academic disputes. However, the medical industry is very different, it is fully controlled by interest groups to achieve revenues. All efforts are directed to what makes money. As long as the medical system is driven by money, medicine must promote flawed research models, foster a drug-reliance culture, and preclude real cures. By failing to cure diseases, the medical industry turns patients into perpetual revenue feeds. Medicine justifies its failure by creating the notion of incurable diseases. This false notion misleads patients to believing that chronic diseases cannot be cured. All those practices make patients to use drugs on a perpetual basis. With time passing, each patient develops a drugrelying habit. When all patients in the nation develop such a habit, controlling symptoms becomes a health culture. All people believe that chronic diseases are incurable and use drugs to control diseases. It is inevitable to form a deeply flawed population health culture. This is why more than half of the national population suffers chronic diseases.

While the drug-for-health culture helps medical industry to generate a great portion of GDP, it extracts maximum pains and suffering from patients. As this system is not able to solve real health problems for three independent reasons I stated, it must result in the worst outcome for the COVID-19 pandemic and hurts everyone. The large number of avoidable deaths, lock-downs, personal isolation and miserable life quality are the costs that all U.S. citizens are paying for this dysfunctional medical system. This system is maintained by drugs developing practices, funding policies, publisher practices, peer reviewers, medical cost reimbursement practice, FDA approval practice, disease treating 
practices, etc. All persons in medicine do their parts to promote drugs, treatment protocols and flawed science. After the system has become a smooth operator, nothing can stop, nobody can stop, and no body wants to stop. Each driving point may be pushed simultaneously by tens to hundreds of persons or organizations, there is no way to stop. Researchers in their articles can only lament noted problems.

Medicine cannot reform and improve itself without outsider force. Congress must take action to change it. To justify interventions, the government should consider expected resistance to changes in any foundation of science. One well known instance is great resistance to the change from the geocentric theory to the heliocentric theory after geocentric theory influenced the world for over 1500 years. The second incidence is elixirs which was believed to make people immortal. Elixirs once gained wide acceptance while minority voice were ignored. In more than two thousand years, elixirs killed a large number of people including at least 12 emperors. Due to the flaws in medicine, drug benefit-to-risk ratios have been grossly exaggerated. I expect that reassessment of drugs by using real science will dramatically lower their benefit-to-risk ratios. It is very possible that synthetic drugs are bad given that their uses clash with every theory I can find and the only thrust is conclusions from clinical trials. When the last ounce of evidence - clinical trial data - is rejected, their uses should be limited to emergence situations.

Congress should be able to understand the problems I have mentioned. My conclusions are backed up by tens of thousands of studies by well recognized researchers. I believe some medical experts know the problem, but none can do anything to change. I thus urge Congress not to rely on experts from medicine and should disregard the consensus of medical experts. The reason can be seen from historical resistance to Quantum Mechanics. When Quantum Mechanics arrived, the majority of classical physicists do not accept it. Classical physicists cannot accept the theory because they are stuck on thinking in classical physics. A good portion of them cannot accept several interpretations in Quantum Mechanics (such as collapse of the wave function, quantum jumps and even existence of particles). Accepting Quantum Mechanics needs acceptance of something that has been taught to be wrong. To reject the foundation of medicine, one must be willing to accept that life is more complex than things they can understand by common sense, is not what one can measure by comparing a treatment with a control, and is not something that can be modeled by mathematical equations.

Medical experts have been stunk with the presumption that everything can be studied by using a clinical trial followed by statistical analysis. However, I found that most life problems including multiple factors causes, mind-body interactions, and the mobility of immune cells in tissues cannot be represented by descriptive language and nor by mathematical equations. The mobility of immune cells is a problem belonging to either classical physics nor Quantum Mechanics. It is a problem no body can understand at this time. Many life 
phenomena cannot be described accurately. No valid method exists to model thinking impulses and their health impacts. This is not a far-fetching belief. Mathematical model cannot be used to evaluate a war or battle. One reason is that most variables are unknown, cannot be defined, or influenced by a large number of unknown and undefined factors. In this respect, personal health is similar to a battle system. However, the human body is far more complex than a battle system.

Disputes in Quantum Mechanics do not directly affect government interest like medicine. Cures cannot be found by looking at potential to make money. Wrong research models mean total waste of research fund, failure to find cures, great pains to patients, and even catastrophes. The toxicity independent action model is one responsible for massive health problems, and is clearly responsible for inability to fix environment, ecosystem, climate, etc. It will be responsible for faster end of civilization. Due to the role of money, researches for correcting the foundation of medicine necessarily meet great resistance from publishers, peer reviewers, researchers, federal agencies (NIH, FDA, CDC, etc), organizations, consumers, and general media. Even academic researchers are inherently biased because no one is willing to see their research findings become junk science. Since medicine affects lives and medical spending, government cannot be a bystander watching resolution of the disputes. Inaction means that medicine will be forever junk science, and humans continues being the slavery of chronic diseases.

\section{F. My Proposed Measures Can Control the COVID-19 Pandemic.}

My attempts in the last year failed to get attention from the research community and government agencies while nearly 400,000 lives were lost. My proposed measures comprise a proactive body temperature management, devices for treating the respiratory track, deep breathing exercise, and systematic renovation of the ventilation systems for homes, office buildings, and public facilities. I have no doubt that those four measures can end the pandemic.

1. Proactive body temperature management is knowledge and thus no body can profit from it. Such knowledge cannot become part of medical practice. It can help 97\% people to avoid infection and death. Most people can learn the skills to avoid infection and death. The skills can help them to resume normal life activities.

2. Promote deep breathing exercise among vulnerable people. I propose this exercise because it is the best measure for fighting lung diseases including COVID-19. In 1950's, a large number of studies were done in China to use various Qigong (a sort of deep breathing exercise) to treat challenging lung diseases. The data acquired at that time is reliable because the studies were not influenced by money. Recently, numerous medical studies show that deep breathing exercise, meditation, relaxation, etc. can promote immune system. 
Some studies show that meditation has great benefits in controlling and mitigating cold and influenza. Given that this line of exercise has been used in multiple cultures for thousands of years, I found that it is far better than any drug. I believe that 3 hours exercise at the right timing can greatly mitigate or even cure a coming infection.

3. I developed a line of medical devices for treating COVID-19 in homes and hospitals. This line of devices would require fund to bring products to the market. Unfortunately, no funding can be funded.

4. The long-term goal of my plan is to improve the ventilation systems for public buildings. The 6 feet distance is an insufficient measure for closed buildings. It is known that influenza virus from animal farms can travel by wind up to 10 miles, and school buildings are full of flu viruses. Six feet distancing only prevents the worse exposure but not a sufficient preventive measure inside closed buildings. One biggest contribution of the high COVID-19 deaths is poor air quality in most working buildings and pubic facilities. To address this problem, I have developed several devices. This is an essential measure for ending this pandemic and reopening schools, universities, and public buildings.

5. The most important measure above all is changes to lifestyle to avoid tens to hundreds of harmful substances. COVID-19 infection, death risk, cancer risk, heart diseases, and AIDS can be attributed to subtle changes in the immune systems. Compromised immune systems are nearly always caused by harmful substances. Avoidance is the only solution. It takes time to see benefits from changes in lifestyle. People need to learn basic skills to avoid exposures to toxic substances.

If those measures are not used, the U.S. cannot do well in controlling the pandemic. Vaccines can provide only partial protection, cannot protect nasal cavities, and may be defeated by viral mutation. Vaccines cannot provide much benefits for fragile people and may by impaired by low temperature. The U.S. cannot bet everything on vaccines and drugs while ignoring personal health and exposures to disease agents.

\section{G. Biased People Preclude My Proposed Pandemic Measures.}

The U.S. has provided a relief package CARES Act to address this pandemic. The package includes $\$ 153.5$ billion of support for public health. I have a clear plan to reduce $97 \%$ deaths, a full plan for reopening businesses, schools and universities, restaurants, etc. Any one would think that the fund would support a research targeting the number-one factor and re-opening schools. However, controlled by flawed medicine and biased persons, the research fund never goes to researches that can put an end to the pandemic. Here are what I have experienced: 
1. I applied for a research fund from NIH, my first rejection is so-called over-stuffing. My application was initially rejected for containing too much writing material. A critical research can be rejected for including one more page. NIH has absurd rules that each application can only have 6 to 12 pages of the research plan. The reason is that all reviewers have the same burden and all applicants can write same amounts. This kind of practices has been in use for decades. The reason is obvious that NIH funded research cannot cure diseases anyway, and thus NIH runs grants like employment benefits.

2. My second application from NIH was rejected because my proposed subject is specifically excluded by the funding opportunity announcement (FOA). My research subject concerns the most important subject: use of lifestyle factors to mitigate infection and death risk. By current NIH practice, NIH specifies a list of things it supports and a list of things it does not. Each FOA contains a large number of limitations as if NIH had already known what would work and what would not. The massive limitations, which range from application size, medical theory and practice, etc. imply that qualified research projects must be population based reductionist, static comparative type. In my view, its specifications guarantee that supported researches repeat failure forever. Moreover, each of the FOA is tailored to research subjects of certain grantees or specific areas that NIH staff believes valid. My lifestyle interventions deal with the most complex issue: disproving use of clinical trials to evaluate performance of intervention. The research directly refutes the comparative method. Moreover, FOA specifies that supported research must use current theories and practices. If the subject of an application is to disapprove existing theories and practices, the application cannot be reviewed. If the existing theory and practice are wrong, NIH keeps supporting failed research forever. By using this kind of practices, NIH never fund a research to challenge existing theories. This is why medicine does not explore temperature. By the action of controlling people, NIH cannot use the fund provided by CARES Act to achieve real benefits for the tax payers.

3. I submitted several pitches to seek seed fund from National Science Foundation (NSF). NSF accepts grant-seeking pitches for COVID-19 only for a few months and then closed. I pitched funding opportunity twice on COVID-19. My research is not directed to revenue-generating objects, naturally the reviewer does not like it. I pitched a second one, I did not win. I developed some marketable products, but my concept clashes with medicine. NSF funding practices are heavily influenced by flawed medicine. Its narrow time window for pitches is a big obstacle. New theories, new inventions and new product concepts often come by strike of luck and could not be planed. NSF practice seems to seek technologies that can be planed like thing from a production line, and pitched within the small time window. NSF would not think how new discoveries are made for the reward of the CARE Act. Its funding practice is not suitable for researches for national health emergence. In addition, word limits like 250-500 would prevent a grant seeker from presenting a full story. Such 
limitation could preclude a subject for challenging medicine. The overall design is to promote same kind of research to achieve the same result.

4. Temperature is responsible for 100 times higher incidence and death rates. The poor pandemic outcome in the U.S. is closely related to the ventilation systems and interactions between lifestyle and ventilation systems. I tried to seek funding from Department of Energy concerning development of technologies for renovating ventilation systems for homes, schools, and universities. This is a radical idea. I was told that the program had expired and I had to wait. The U.S. has missed one year without doing anything about ventilation systems in homes, schools, universities, office buildings, and restaurants. Solution to this problem is not easy. If the U.S. wants to end the day of wearing mask, this is an essential measure. Social distancing does not help much in most situations and is often misused to cause much worse results. While DE program could not be regarded as relevant to COVID-19, it is not entirely surprising. My first article stresses air ventilation as a key pandemic measure. Medicine does not do anything about it.

5. What is far worse is that each controlling person or entity uses its power to prevent, preclude and suppress disruptive discoveries or anything outside preferred options. For example, the National Library of Medicine (NLM) accepts only articles that are supported by NIH or published by a commercial publisher as a publish-for-money trade. Essentially, NLM excludes anything that belongs to flawed medicine. Even worse, medical publishers charge the same fees for articles done solely for pubic benefits and marketing articles promoting drug revenues. When medicine has lost the sense of health justice, the U.S. cannot control the pandemic, could not have saved 400,000 lives, cannot address the health crisis, and will never solve the on-going medical spending crisis.

Model flaws, narrow pandemic options, professional biases, incompetence, laziness, deception, fraudulent intention, etc. are the reasons for societal failure to end the pandemic. If the ventilation problem is not addressed, schools classrooms, restaurants, office buildings will not be safe places. Even after this pandemic ends, the U.S. will face similarly poor pandemic outcomes in future. While I can keep wait, more lives will be lost, society repeats lock-down and people continue living their life in isolation.

What is responsible for the bad pandemic outcome is the people who manage federal funds. The Public Health Service Act of Title 42 is promoting national health and addressing national health crisis. The law does not say that it can only be achieved by using this deeply flawed medicine. Even if Congress had accepted the science-based medicine in the past, Congress must have assumed that science-based medicine is the valid and best. However, I have proven that medicine is deeply flawed in all aspects, driven by money and preclude cures. Congress would not have invested trillion of tax dollars to a medicine that cannot deliver cures. Even if medicine were valid, treatment options are limited by interest groups to promote the less than $1 \%$ of options. Congress would not have accepted the medicine as exclusive remedies. Even if the model flaws and 
treatment biases did not exist, most health problems cannot be addressed by medical treatments. Congress would not have limited the fund to failed researches that will fail forever. The most effective pandemic measure is to improve human immune system. Many measures must be directed to cooking habits, food production lines, air and water.... When a person is loaded with heavy metals, carcinogens and immune suppressants, the immune system opens its door to COVID-19, HIV, cancer, heart diseases, etc. There are hundreds of ways to promote personal immunity, but medicine promotes NONE. If medicine has been inclusive like a real science, most spending in the Affordable Care Act would have vanished long ago.

Congress should establish a Commission to prove every point I state above. Congress should establish an independent agency to seek, discover and disseminate knowledge of true health science. Like HIV, cancer, and heart diseases, the most effective solutions to the pandemic is lifestyle changes, air quality, health wisdom, exercise, and personal skill to avoid massive toxic substances in daily life activities. If the U.S. continues supporting medicalization with drugs, the U.S. will repeat failure perpetually and will face a much worse health crisis.

\section{H. A Plan to Find Cures in Ten Years and End the COVID-19 Pandemic in Two Years.}

\section{Flaws in Medicine Cannot Be Corrected.}

Medicine fails for centuries as a result of using flawed presumptions in its foundation. Such medicine fails to find cures for cancer, heart disease, AIDS, infertility, mental diseases, etc. The overall performance in chronic diseases is often less than $1 \%$ with a few percents being the highest. All chronic diseases are officially regarded as incurable but treatable. Medical treatments are deeply flawed in the foundation of medicine, the selection of treatment options, manner of addressing disease cause factors, and the teaching of incurable diseases. Its worse roles are precluding all real cures in the remaining 99\% options, has fostered deeply flawed health culture and ruined people health wisdom. Naturally, medicine is unable to use the strongest factor to conquer the pandemic, cannot save 30 million annual premature deaths in chronic diseases, and will not able to to end the pandemic in the most effect manner. Every life can end by diagnosis of a terminal disease.

Congress cannot hope that medicine can reform itself. Congress should consider how large reforms occurred. Resistance to Quantum Mechanics arises from people's inability to understand. Articles on Quantum Mechanics can be published because non-medical publishers are not controlled by interest groups. Much bigger resistance to medical reform comes from interest groups. I sent a large number of invites to medical experts in universities and medical schools, but I did not get one to review, comment and support my article. Even though 
correcting flaws in medicine will benefit them personally, they cannot do one single thing. It took more than a thousand years to change the geocentric theory. It took a century to just note problems in somatic mutation theory. Researchers cannot correct scientific flaws without centuries of delay. If a system is controlled by money, the system cannot be reformed by internal force.

Medicine is a unique field that is highly regulated by law. After decades of its existence, interest groups have found ways to run and control medicine. This is why a foundational flaw in medicine cannot be fixed by correcting errors only. All errors and flaws have found their ways into grant practices, publishing practices, drug-approving practices, marketing practices, medical practices, medical cost reimbursing practices, insurance practice, etc. Model flaws, narrow treatment options, and intent to kill cures can be found in all aspects of medicine. It is natural that NIH uses flawed presumptions, flawed models, flawed theories, flawed practices, etc. to administrate research fund. NIH funding practices clearly reveal that NIH misplaces its focus to applicant's right and peer review burdens with no real intent to find cures.

All strands in the medical system have been tainted by model flaws, option biases, exclusionary intent, fraudulent intent, etc. No one can do anything to change such system. My first article on clinical trial was posted more than a year ago, but medical experts keep silence as if the article did not exit, while medical publishers including Nature, Science, Lancet, etc. refused to review my article. Dishonest editors could not provide a single reason but just frivolously stated that my article does not fit in their journals. Since my article refutes the foundation of medicine, the article fits nowhere. Their silence is clear evidence that they cannot defend, and their refusal reflects their scientific dishonesty and money-driven bias.

\section{Establish Federal Health Science Commission (FHSC).}

I urge Congress to establish an independent federal agency which may be referred as Federal Health Science Commission (FHSC). The purpose of the Commission is promoting public health and addressing national health crisis. Congress should disband half of NIH funding programs, use remaining fund to support research in basic science, acute diseases and emergency medicine, and relocate the other half of findings to the FHSC. The mission is to find cures for chronic disease within 10 years and has the incurable term disappeared from all medical literature and general media. Supported health measures must cover everything under the Sun. Some missions are as follows:

(1) Support researches to explore flaws in medicine, and develop and improve research models and practicing models. The research is not to support what is in the medical books, but to find evidence to disprove existing theories and practices and find alternatives. Funded researches are exposing the limitations and flaws in medical research models, disease theories, current medical practices, etc. One main mission is establishing mechanisms to disrupt vested interests. 
(2) Correct public health wisdom so that people will understand that health cannot be achieved by synthetic drugs. Establishing a health culture is the key for U.S. future success. The population should be educated with the knowledge that drugs are the last options. They are used only to save life and prevent imminent death.

(3) Support and fund researches to explore cancer miracles and other health miracles. The fastest path is to find cures by exploring successful cases.

(4) Support researches for understanding disease risks that come from kitchens, food production line, working environment, and disease-causing lifestyle factors. I estimate that most diseases can be prevented by proactive attempts to avoid exposures to disease-causing substances. It is possible that one bad product or one single cooking practice may make millions of sick people.

(5) Provide public health database for promoting public health. Private marketing has completely ruined public health wisdom. The reliance by population on wrong knowledge is responsible for massive future medical spending. Government should provide its own media service to counteract misleading commercial messages. Government must disseminate its own health education materials for the public.

(6) Develop regulations to prohibit misleading and fraudulent market practices. It is time to ban commercial publishers from claiming scientific validity unless the publishers are open to everything under the Sun. Any restrictions set to preferentially promote flawed articles should be the basis for ban.

(7) Encourage patient discoveries and folk remedies. True heath art is far more complex than any other science due to life phenomenon and multiple factors interactions. Knowledge of personal body is not what every peer can see or understand. Change peer review role and abandon the general acceptance standard, but adopt non-obvious standard as a supplemental criterion for evaluating complex research issues.

(8) Develop lifestyle prescription method and exercise prescription method. The Commission should promote future lifestyle prescription as part of medical practice, and promotes exercise as the primary cures. Future research should be encouraged to explore exercise type, duration and intensity, and disprove the flawed notion that 15 minutes of daily exercise could do the job.

(9) I believe that SARS-CoV-2 is nature's revenge against human activities of ruining environment, ecosystem and climate. There are obvious connections between AIDS, potentially this SARS-CoV-2 AIDS, and more future AIDS in light of environment, ecosystem and climate. Climate with extreme temperature, and large temperature fluctuations is an important contributory factor. After human bodies are loaded with toxic substances, humans loses immunity against diseases.

\section{Selection of Staff}


Selection of staff will be the key for its success. As indicated by the historical problems in each ground-breaking discoveries, people in the old camp will not support changes. The staff composition should strictly limit the people trained in classical medical schools. Potential training background include systems biology, integrative biology, systems microbiology, systems ecology, systems medicine, integrative medicine, integrative systems toxicology, system genetics, neuroscience, mathematicians, physicists and chemists, system dynamics, occupation safety, building construction, food nutrition, food processing and science, exercises and sports, environmental protection, etc. Many of those fields of science stress the whole system. Diseases come from diet, air, environment, kitchens, buildings, working places, thinking habits, and working habits, etc.

\section{Ban Fraudulent Marking}

Medicine contains massive obsolete, incorrect, and wrong knowledge. Some knowledge is clearly wrong; some knowledge is subjects of debates; and some knowledge is right. Most findings in research articles are right for some use purposes but wrong in other use purposes. Marketing practice should be regulated to prevent interest groups from using wrong and obsolete science to profit at the expense of patient lives, population health, and national economy. Since many issues may be in dispute, the FHSC should decide what is permissible and what is not.

To prevent interest groups from using flawed science to promote inferior treatments at expense of real cures, a rule should be established to separate professional art from true medical science. This division allows anyone to advertise according to their interests. The marketing of drug companies and practicing doctors should be classified as professional art. Medical science must be discovered by persons, patients, and research groups without support from interest groups. Researches on patented objects and trademarked services may not be claimed as health science. To qualify as health science, covered objects and services must be one that can be replaced by other choices. Details are provided to bar companies from making cross-promotion attempts. Law should prevent interest groups from finding ways to defeat the ban. Allowing money to influence the selection of medical options will repeat the failure of medicine. Criminal punishment should be placed to punish illegal conduct.

\section{Establish Fast Legislative Oversight Mechanisms}

I anticipate that anyone may say that ten year time window to find cures for chronic diseases is infeasible. I note that there is sufficient knowledge for addressing chronic diseases. The tens of thousands of disease causes articles point to right directions. My plan is to utilize researches in the last half century. Based on well reasoned studies, I must find that cancer, and heart disease are solved among most cases, but real difficulty is using right methods to cure diseases. AIDS researches have discovered a great deal of the knowledge, but may require more studies. I also believe COVID-19 is a problem that can be 
addressed by several things or lifestyle change. In addition, there was no blank incurable notion in ancient medicines. Moreover, there are a large number of disease self-resolution cases. There are hundreds of cases reflecting tumor selfresolution and I believe there are millions of unreported cases. I knew of two personal cases: one patient died of "cancer" one day before his (negative) diagnostic report was issued; and in another case, a terminal cancer patient survived for more than 30 years after he had been told that the tumor had been completely removed (In fact, people close to the surgeon knew that tumor was touched). It is clear that diseases came and go, depending on the feeling of the immune system. There are hundreds of ways to improve immune systems.

The question is not whether diseases can be cured, but how fast a right approach will be used as primary measures for chronic diseases. My proposed plan is to use the legislative process, and the overall time would depend on how fast the legislation can be enacted and how fast Congress can amend its law. In bringing the new health science, I expect that many disputes will appear. If all legal disputes are disposed by court in the traditional docket clocks, two major sequential legal actions would consume ten years. If the reform takes 50 years to complete, most of people in our time will not see the fruit of reform and young people will become victims of the flawed and exclusionary medicine. Damages to their health can never be undone; and damages caused by exposure to toxic substances will hurt them for their lives.

To speed up the health science reform, a newly enacted legislation should include a fast-track dispute resolution mechanism. One component of law requires the court to decide any health science case as soon as possible. Since court is not a good institution to address complex health problems, Congress should authorize the Commission to establish regulations and interpretive rules. In addition, the new legislation should include a clause to allow the Commission to send draft regulations and interpretive rules for Congress's enactment on a fast track. If the delay by the legal process is eliminated, ten years is a feasible for most chronic diseases.

Medicine fails because it is deeply flawed in its foundation. Medicine cannot improve itself because it is controlled by money. To learn from the lesson, Congress must include strict conflict-of-interest rules. All voting staff of FHSC may not have any financial interest and professional interest in its decisions. If the staff owns no corporate stock, get no practicing revenue, receive no publishing revenue, or get no indirect gain, the staff would have undivided interest for patients, national economy and mankind. Their decisions will affect every person including themselves. The Commission can achieve the intended purpose of bringing cures to the world. However, if the FHSC is controlled by money, the reform will produce a second flawed medicine and a duplicate dysfunctional system.

The U.S. has an opportunity to bring cures to the world, control the COVID19 pandemic in two years, mitigate trillions dollars of loss in economy, and save trillions dollars of medical spending. Radical medical reform is the only realistic 
measure for solving medical spending shortage. Nothing can solve medical spending problems unless medicine can cure diseases. The reform will save 30 million premature lives in the world each year. By creating true health science, the U.S. can lead the world again. Failure to take action means disasters to mankind and massive avoidable deaths. It is a clear call for both democratic and republic lawmakers. The benefits are for all human beings. Diseases have killed the rich, the poor, the young and the old. True health science should never like the failed medicine controlled by money. 